\title{
Linear and nonlinear piezoelectric shunting strategies for vibration mitigation
}

\author{
P. Soltani ${ }^{1, \text { a }}$, G. Tondreau ${ }^{2}$, A. Deraemaeker ${ }^{2}$, G. Kerschen ${ }^{1}$ \\ 1 Space Structures and Systems Laboratory, Dept. of Aerospace and Mechanical Engineering, University of Liège, Belgium \\ 2 Building Architecture and Town Planning (BATir) Avenue F.D. Roosevelt 50, Brussels, B-1050, Belgium
}

\begin{abstract}
This paper studies linear and nonlinear piezoelectric vibration absorbers that are designed based on the equal-peak method. A comparison between the performance of linear mechanical and electrical tuned vibration absorbers coupled to a linear oscillator is first performed. Nonlinearity is then introduced in the primary oscillator to which a new nonlinear electrical tuned vibration absorber is attached. Despite the frequency-energy dependence of nonlinear oscillations, we show that the nonlinear absorber is capable of effectively mitigating the vibrations of the nonlinear primary system in a large range of forcing amplitudes.
\end{abstract}

\section{Introduction}

Tuned vibration absorbers (TVAs) are passive devices that allow to reduce the vibration of a host structure in a specific frequency band. An electrical TVA (ETVA) consists of a resonant electrical shunt in series with a piezoelectric (PZT) material attached to the host structure. Dynamical coupling between the host and the PZT converts mechanical energy into electrical energy. This electrical energy is then dissipated by the resistor of the shunt which causes the vibration of the host system to be mitigated. In other words, ETVAs dissipate energy similarly to mechanical TVAs (MTVAs) but without adding any moving part to the host structure. Hagood and von Flotow [1] proposed an analytical model of a linear ETVA, and this model was used later by Preumont et al. [2]. A nonlinear ETVA was introduced in [3], and the effects of nonlinear electrical shunts on linear host structures were explained analytically using a perturbation method.

The objective of the present paper is to revisit the classical equal-peak tuning rule when it is applied to both linear and nonlinear ETVAs. The paper is organized as follows. A comparison between the mitigating performance of a linear MTVA and a linear ETVA is first presented in Section 2. Then, Section 3 discusses how vibration mitigation of a nonlinear host structure can be improved by considering a nonlinear ETVA. Conclusions of this study are drawn in Section 4.

\section{Linear piezoelectric tuned vibration absorbers}

A simplified model of a host structure with mass $M_{1}$, damping $B_{1}$ and stiffness $K_{1}$ is considered herein. The structure is excited by an external harmonic force of amplitude $F_{0}$ and frequency $\omega$. A PZT shunt is attached to this mechanical oscillator, as illustrated in Fig. 1.

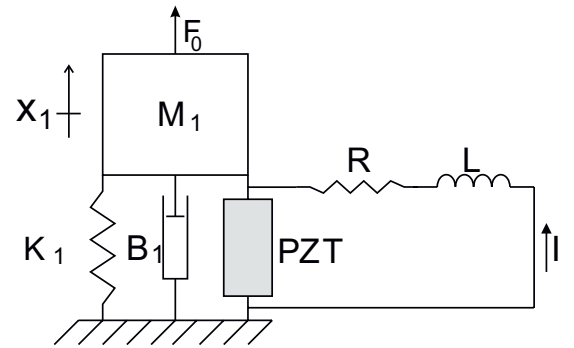

Fig. 1. Piezoelectric vibration absorber with a (series) RL shunt.

The PZT shunt consists of a PZT rod with open-circuit stiffness $K_{P Z T}^{*}$ and capacitance $C^{S}$ (under constant strain) attached to a resonant series resistive-inductive (RL) shunt. The steady-state vibration amplitude $X_{1}$ and the charge in the PZT shunt $Q$ are the degrees of freedom of this model. The governing equations of motion of this system are:

$$
\begin{aligned}
& M_{1} \ddot{X}_{1}+B_{1} \dot{X}_{1}+\left(K_{1}+K_{P Z T}^{*}\right) X_{1}-H Q=F_{0} \sin \omega t \\
& L \ddot{Q}+R \dot{Q}+\frac{1}{C^{S}} Q-H X_{1}=0
\end{aligned}
$$

The parameter $H$ is the piezoelectric constant in the d33mode (axial mode), as explained in [2]. The dimensionless form of Eq. (1) can be written as:

$$
\begin{aligned}
& {\left[\begin{array}{ll}
1 & 0 \\
0 & 1
\end{array}\right]\left\{\begin{array}{l}
x_{1,}^{\prime \prime} \\
q^{\prime \prime}
\end{array}\right\}+\left[\begin{array}{cc}
2 \mu & 0 \\
0 & r \delta^{2}
\end{array}\right]\left\{\begin{array}{l}
x_{1}^{\prime} \\
q
\end{array}\right\}} \\
& +\left[\begin{array}{cc}
1 & -\alpha_{1} \delta \\
-\alpha_{1} \delta & \delta^{2}
\end{array}\right]\left\{\begin{array}{c}
x_{1} \\
q
\end{array}\right\}=\left\{\begin{array}{c}
f_{0} \sin \gamma \tau \\
0
\end{array}\right\},
\end{aligned}
$$

where the prime stands for the derivative with respect to $\tau$ and where the dimensionless parameters are defined in a similar way as in [3]:

$$
\begin{array}{ll}
\Omega_{1}=\sqrt{\frac{K_{1}+K_{P Z T}^{*}}{M_{1}}}, & \Omega_{e}=\frac{1}{\sqrt{L C^{S}}}, \quad \delta=\frac{\Omega_{e}}{\Omega_{1}}, \\
\tau=t \Omega_{1}, & \mu=\frac{B_{1}}{2 M_{1} \Omega_{1}}, \quad r=R C^{S} \Omega_{1}, \\
q=\sqrt{L} Q, & x_{1}=\sqrt{M_{1}} X_{1}, \gamma=\frac{\omega}{\Omega_{1}}, \\
\alpha_{1}=\sqrt{\frac{k^{2}}{1-k^{2}}} \sqrt{\frac{K_{P Z T}}{K_{P Z T}^{*}+K_{1}}}, K_{P Z T}^{*}=\frac{K_{P Z T}}{1-k^{2}}, & f_{0}=\frac{F_{0}}{\Omega_{1} \sqrt{K_{1}+K_{P Z T}^{*}}},
\end{array}
$$

a e-mail: payam.soltani@ulg.ac.be 
where $K_{P Z T}$ is the short-circuit stiffness, and $k$ is the electromechanical coupling factor.

The transfer function of the primary oscillator $G_{E T V A}(\gamma)=$ $\frac{x_{1}}{f_{0}}$ can be expressed as:

$G_{E T V A}(\gamma)=\left|\frac{j \delta^{2} r \gamma+\delta^{2}-\gamma^{2}}{\gamma^{4}-j \delta^{2} r \gamma^{3}-\left(\delta^{2}+1\right) \gamma^{2}+j \delta^{2} r \gamma+\left(1-\alpha_{1}^{2}\right) \delta^{2}}\right|$

\subsection{Equal-peak method applied to MTVAs and ETVAs}

The MTVA is a widely-used passive vibration damping device. It consists of a secondary mass $M_{2}$ connected to the host structure through a spring $K_{2}$ and a dashpot $B_{2}$, as shown in Fig. 2.

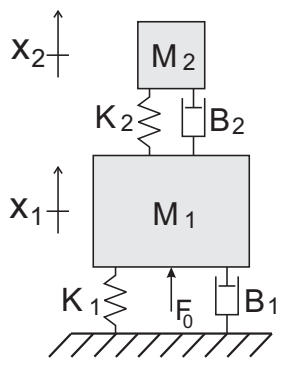

Fig. 2. Mechanical tuned vibration absorber (MTVA).

Considering a harmonic excitation, the equations of motion are:

$$
\begin{aligned}
& M_{1} \ddot{X}_{1}+B_{2}\left(\dot{X}_{1}-\dot{X}_{2}\right)+B_{1} \dot{X}_{1}+K_{2}\left(X_{1}-X_{2}\right)+K_{1} X_{1} \\
& =F_{0} \sin \omega t \\
& M_{2} \ddot{X}_{2}+B_{2}\left(\dot{X}_{2}-\dot{X}_{1}\right)+K_{2}\left(X_{2}-X_{1}\right)=0
\end{aligned}
$$

As for the ETVA, it is more convenient to express Eq. (5) in terms of dimensionless parameters. If one defines the mechanical tuning parameter $\delta_{m}=\frac{\omega_{2}}{\omega_{1}}\left(\omega_{i}=\sqrt{\frac{K_{i}}{M_{i}}}\right.$ with $i=1,2)$, the mass ratio $\beta=\frac{M_{2}}{M_{1}}, \xi_{i}=\frac{B_{i}}{2 \sqrt{K_{i} M_{i}}}$ the modal damping ratio of the host $(i=1)$ and the MTVA $(i=2)$ respectively, and $x_{i}=\frac{X_{i}}{\sqrt{M_{i}}}$, Eq. (5) becomes:

$$
\begin{aligned}
& {\left[\begin{array}{ll}
1 & 0 \\
0 & 1
\end{array}\right]\left\{\begin{array}{l}
x_{1}^{\prime \prime} \\
x_{2}^{\prime \prime}
\end{array}\right\}+\left[\begin{array}{cc}
2\left(\xi_{1}+\beta \delta_{m} \xi_{2}\right) & -2 \sqrt{\beta} \delta_{m} \xi_{2} \\
-2 \sqrt{\beta} \delta_{m} \xi_{2} & 2 \delta_{m} \xi_{2}
\end{array}\right]\left\{\begin{array}{l}
x_{1}^{\prime} \\
x_{2}^{\prime}
\end{array}\right\}} \\
& +\left[\begin{array}{cc}
1+\delta_{m}^{2} \beta-\sqrt{\beta} \delta_{m}^{2} \\
-\sqrt{\beta} \delta_{m}^{2} & \delta_{m}^{2}
\end{array}\right]\left\{\begin{array}{l}
x_{1} \\
x_{2}
\end{array}\right\}=\left\{\begin{array}{c}
f_{0} \sin \gamma \tau \\
0
\end{array}\right\}
\end{aligned}
$$

The receptance function of the primary mass $G_{M T V A}(\gamma)=$ $\frac{x_{1}}{f_{0}}$ therefore reads:

$$
\begin{aligned}
& G_{M T V A}(\gamma)= \\
& \left|\frac{2 j \delta_{m} \xi_{2} \gamma-\gamma^{2}+\delta_{m}^{2}}{\gamma^{4}-2 j \delta_{m} \xi_{2}(1+\beta) \gamma^{3}-\left(1+(1+\beta) \delta_{m}^{2}\right) \gamma^{2}+2 j \gamma \delta_{m} \xi_{2}+\delta_{m}^{2}}\right|
\end{aligned}
$$

Den Hartog proposed a constructive method for the tuning of MTVAs [4], which is still widely used today. Because the receptance function passes through two invariant points that are independent of absorber damping, he proposed (i) to adjust the absorber stiffness to have two fixed points of equal heights, and (ii) to select the absorber damping which is the average of the two damping values that realize a horizontal tangent through the left and right fixed points, respectively. This tuning condition minimizes the maximum response amplitude of the primary system and lays down the foundations of the so-called equal-peak method. Assuming an undamped host structure (i.e., $B_{1}=$ $0)$, Den Hartog [4] and Brock [5] derived approximate analytic formulas for the absorber stiffness and damping, respectively:

$$
\delta_{m, o p t}=\frac{1}{1+\beta} \quad \text { and } \quad \xi_{2, o p t}=\sqrt{\frac{3 \beta}{8(1+\beta)}}
$$

Interestingly, it is only recently that an exact closed-form solution to this classic problem could be found [6].

Hagood and von Flotow proposed an extension of this method to the ETVA in [1]. The design process can be summarized as follows:

1. A PZT and an inductance $L$ are chosen so that $\delta=$ $\delta_{H V F}=1$, which means that the electrical natural frequency $\Omega_{e}$ should be the same as the host structure natural frequency $\Omega_{1}$. This first design rule ensures that the fixed points have the same amplitude.

2. A resistor value is chosen so that $r=r_{H V F}=\sqrt{2} \alpha_{1}$. This second design rule consists in setting the amplitude of $G_{E T V A}(\gamma)$ at $\gamma=\delta_{H V F}=1$ to the amplitude of the fixed points. Doing so, the two peaks in the receptance curve have approximately the same amplitude, and their maxima are very close to the fixed points.

However, it turns out that this design rule for the ETVA is not in exact correspondence with Den Hartog's rule, as discussed in the next section.

\subsection{Equivalence between MTVAs and ETVAs}

The similarities between MTVAs and ETVAs are investigated in this section through the direct comparison of Eqs. (2) and (6) for $B_{1}=0$, i.e., for $\mu=\xi_{1}=0$. If the mass matrices of these two systems are identical (unitary matrices), the damping and stiffness matrices are only identical if $\beta=0$ and $\alpha_{1}=0$, i.e., if there is no mass $M_{2}$ and no shunt, respectively. This is an obvious result, because the system reduces to the primary oscillator in both cases.

However, it is possible to design an ETVA which is similar to a MTVA by considering small values of $\beta$ and $\alpha_{1}$. Additional conditions to have similar stiffness and damping matrices are (i) $\delta \approx \delta_{m}$, (ii) $\alpha_{1}=\sqrt{\beta} \delta_{m}$ and (iii) $r=2 \xi_{2} / \delta_{m}$. The first of these three equivalence conditions requires that $\Omega_{e} \approx \omega_{2}$; this means that the electrical shunt should be tuned at the same frequency than the MTVA. The second condition links the coupling factor of the PZT in the ETVA to the mass ratio $\beta$ in the MTVA. Note that using Hagood's tuning rule for the ETVA $\left(\delta_{H V F}=1\right)$ and Den Hartog's tuning rule for the MTVA $\left(\delta_{m, o p t}=\frac{1}{1+\beta}\right)$ is compatible with condition (i) for small $\beta$.

In order to illustrate this similarity between the ETVA and MTVA, a numerical example is considered. In this application, a mass $M_{1}$ of $1 \mathrm{~kg}$ is attached to a spring $K_{1}$ much stiffer than the piezoelectric stack used in the ETVA (PZT 
rod with $10 \mathrm{~mm}$ of diameter and a thickness of $100 \mathrm{~mm}$ ), i.e., $K_{1}=\kappa K_{P Z T}$ with $\kappa=100$. If the PZT is a MorganPZT806 [7], $K_{P Z T}=53,42 \times 10^{6} \mathrm{~N} / \mathrm{m}, C^{S}=4,505 \mathrm{pF}$ and $k=0,694$. Figure 3 compares the receptance of this ETVA tuned using Hagood's tuning rule $\left(\delta_{H V F}=1\right)$ for different values of $r$ :

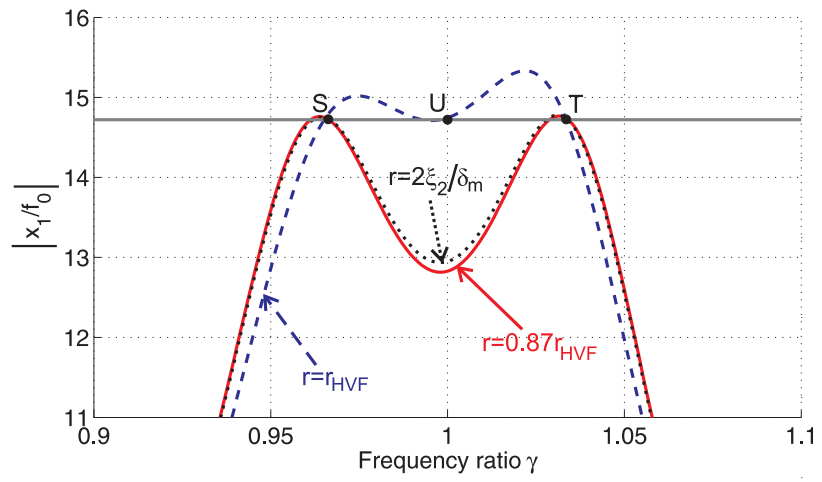

Fig. 3. $G_{E T V A}(\gamma)$ for different values of $r(\delta=1)$.

As already discussed, considering Hagood's tuning rule $r=r_{H V F}$ consists in setting the amplitude of $G_{E T V A}$ at $\gamma=\delta=1$ (i.e., point $U$ ) and at the fixed points (i.e., points $S$ and $T$ ) to the same value. These three points are therefore aligned along the grey solid horizontal line in Figure 3. Clearly, this tuning rule does not provide equal peaks in the receptance curve. By trial and error, it was found that $r=0.87 r_{H V F}$ leads to a frequency response with two equal peaks, which, in turn, gives improved performance in terms of amplitude reduction. It is interesting to observe that designing the ETVA using the third equivalence condition between the MTVA and the ETVA, $r=2 \xi_{2} / \delta_{m}$, generates a frequency response almost identical to the equalpeak design found for $r=0.87 r_{H V F}$.

Figure 4 depicts the frequency response of the primary mass with an attached MTVA tuned using Eqs. (8) and an attached ETVA tuned according to $\delta=1$ and $r=0.87 r_{H V F}$. The fixed points $P$ and $Q$ of the MTVA are shown together with their counterparts $S$ and $T$ for the ETVA.

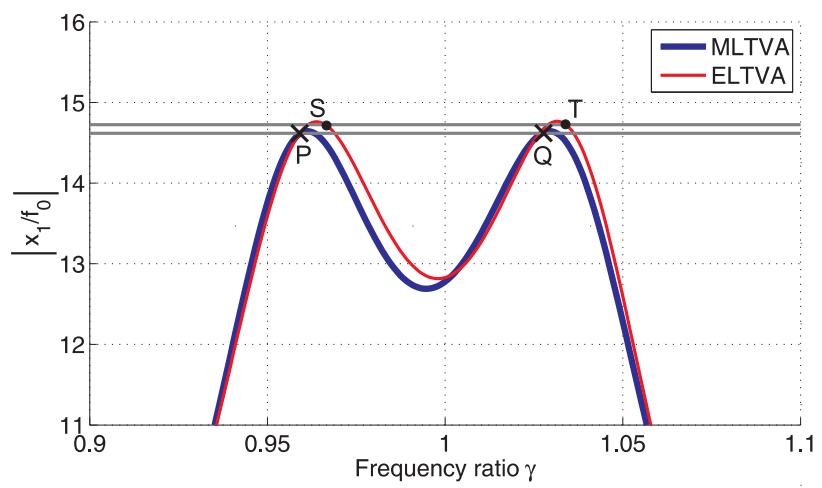

Fig. 4. Frequency response function of a primary mass with an attached MTVA or ETVA (equal-peak design).

Figure 4 confirms that the equivalence conditions lead to an ETVA and a MTVA with similar, but not identical, performance. Specifially, the frequencies of the two peaks of $G_{E T V A}$ are slightly higher than the frequencies of the peaks of $G_{M T V A}$. This is due to the fact that the equivalence conditions neglect the stiffening effect of the host due to the PZT in the ETVA. The present investigations allow us to conclude that, for small $\alpha_{1}$ (this implies large values of $\kappa$ ) and small $\beta$, which is typically the case in real applications, an ETVA can be designed to provide performance very close to the optimal performance of a MTVA.

\subsection{ETVA attached to a nonlinear primary structure}

The performance of a linear ETVA in nonlinear regimes of motion is now investigated by adding a cubic spring $K_{N L}$ in the primary system:

$$
\begin{aligned}
& M_{1} \ddot{X}_{1}+B_{1} \dot{X}_{1}+\left(K_{1}+K_{P Z T}^{*}\right) X_{1}+K_{N L} X_{1}^{3}-H Q=F_{0} \sin \omega t \\
& L \ddot{Q}+R \dot{Q}+\frac{1}{C^{S}} Q-H X_{1}=0
\end{aligned}
$$

The dimensionless parameter of the nonlinear spring is $k_{n l}=$ $\frac{\Omega_{1}^{2}}{\left(K_{1}+K_{P Z T}^{*}\right)^{2}} K_{N L}$, and $k_{n l}=0.000151$ is chosen herein.

The frequency response of the primary mass is plotted in Figure 5 for low and high forcing amplitudes corresponding to linear and nonlinear regimes of motion, respectively. This figure indicates that the ETVA is no longer effective when nonlinearity is activated due to the important difference in the amplitude of the two resonance peaks. A hardening behavior characteristic of cubic springs with positive coefficients can also be observed in the second resonance peak. Clearly, in view of the frequency-energy dependence of nonlinear oscillations and of the narrow bandwidth of the linear ETVA, this absorber can only be effective in linear or weakly nonlinear regimes of motion.

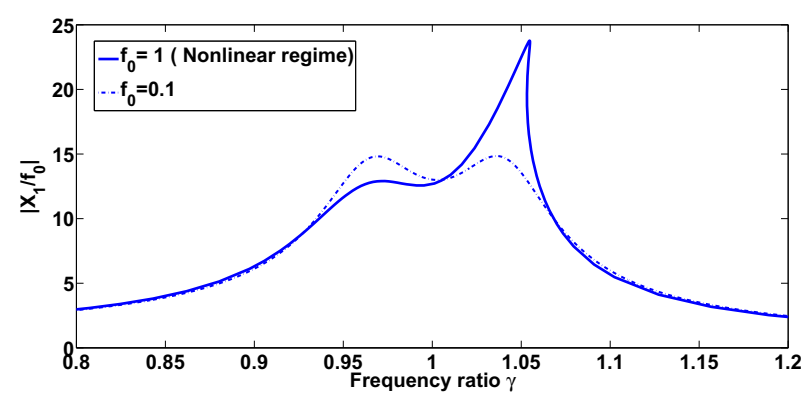

Fig. 5. Frequency response of a nonlinear primary structure with an attached linear ETVA.

\section{Nonlinear piezoelectric tuned vibration absorbers}

In view of the results presented in Section 2.3, it is meaningful to examine the performance of nonlinear piezoelectric tuned vibration absorbers for vibration mitigation of nonlinear primary structures. The nonlinearity can be implemented using the nonlinear capacitance of the piezoceramic or by adding an extra nonlinear capacitance to the shunt [3]. The latter case is considered herein, and a cubic nonlinear term $A_{N L} Q^{3}$, with a corresponding dimensionless parameter $\alpha_{n l}=\left(C^{S}\right)^{2} \delta^{2} \Omega_{e}^{2} A_{n l}$, is added to the shunt 
equation of the system:

$$
\begin{aligned}
& M_{1} \ddot{X}_{1}+B_{1} \dot{X}_{1}+\left(K_{1}+K_{P Z T}^{*}\right) X_{1}+K_{N L} X_{1}^{3} \\
& \quad-H Q=F_{0} \sin \omega t \\
& L \ddot{Q}+R \dot{Q}+\frac{1}{C^{s}} Q+A_{N L} Q^{3}-H X_{1}=0
\end{aligned}
$$

We note that the choice of the functional form of the nonlinear capacitance is important. It can be shown that good performance is obtained when the same functional form as that of the primary system is chosen.

Once the PZT is chosen, it remains to determine $\delta, r$ and $\alpha_{n l}$. To ensure satisfactory performance at low forcing amplitudes for which the primary system behaves almost linearly, $\delta$ and $r$ are determined according to the linear rule presented in the previous section, i.e., $\delta=1$ and $r=2 \xi_{2} / \delta$. The coefficient $\alpha_{n l}$ is then chosen to enforce equal peaks in nonlinear regimes of motion. For $f_{0}=1$, Figure 6 illustrates that equal peaks are achieved when $\alpha_{n l}=3 \times 10^{-4}$. The corresponding receptance curve represents a significant improvement over the receptance curve in Figure 5.

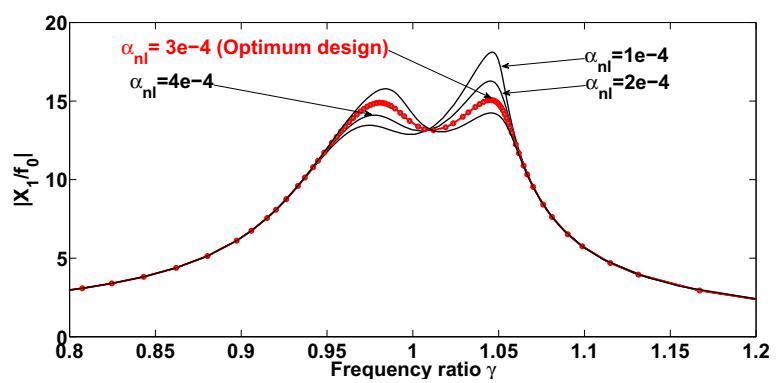

Fig. 6. Frequency response of a nonlinear primary structure with an attached nonlinear ETVA $\left(f_{0}=1\right)$.

To investigate the robustness of the design of the nonlinear ETVA, the frequency response of the nonlinear host structure is calculated for various forcing amplitudes $f_{0}$. Figure 7 shows that equal peaks are realized from $f_{0}=0.3$ to $f_{0}=1.2$, which is an appealing result. From $f_{0}=1.2$, the second resonance peak is characterized by a slightly larger amplitude than the first peak. When $f_{0}=1.5$, the detuning is much more pronounced, and, beyond $f_{0}=1.7$ (not shown here), the nonlinear ETVA gets strongly detuned due to merging of a detached resonance curve with the second resonance peak. The detailed description of this mechanism is beyond the scope of this paper. Another interesting observation is that the amplitude of the resonance peaks does not change substantially when $f_{0}$ increases, which means that the response of the coupled system is almost proportional to the forcing amplitude, as it would be the case for a linear system.

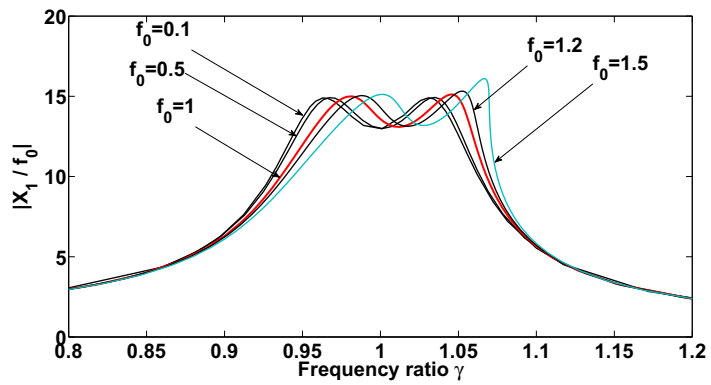

Fig. 7. Frequency response of a nonlinear primary structure with an attached nonlinear ETVA for various forcing amplitudes $f_{0}$.

\section{Concluding remarks}

The objective of this paper is to analyze the performance of linear and nonlinear piezoelectric shunts when they are attached to linear and nonlinear primary systems. In the completely linear case, it was shown that the tuning rule proposed by Hagood and von Flotow does not exactly lead to an equal-peak design. To reach this optimum design, an improvement of the tuning rule was proposed and validated numerically. It was also demonstrated that equal peaks can be maintained in a relatively large range of forcing amplitudes when a nonlinear ETVA is attached to a nonlinear primary system. These preliminary results are encouraging for the further development of the concept of nonlinear ETVA.

\section{Acknowledgments}

All authors, P. Soltani, G. Tondreau, A. Deraemaeker and G. Kerschen, would like to acknowledge the financial support of the Belgian National Science Foundation FRS-FNRS (PDR T.0028.13). The author G. Kerschen would like to acknowledge the financial support of the European Union (ERC Starting Grant NoVib 307265).

\section{References}

1. N.W. Hagood, N.W. and A. von Flotow, Damping of structural vibrations with piezoelectric materials and passive electrical networks. Journal of Sound and Vibration, 146, pp. 243-268, 1991.

2. A. Preumont et al., The damping of a truss structure with a piezoelectric transducer. Computers and structures, 86, pp. 227-239, 2008.

3. G.S. Agnes and D.J. Inman, Nonlinear piezoelectric vibration absorbers. Smart materials and structures, 5, pp. 704-714, $1996 .$.

4. J.P. Den Hartog, Mechanical vibrations (Dover Publications, New York 1985) pp. 93-104.

5. J.E. Brock, A note on the damped vibration absorber. Journal of Applied Mechanics, 68, pp.A-286, 1946.

6. T. Asami and O. Nishihara, Closed-form exact solution to $H_{\infty}$ optimization of dynamic vibration absorbers (application to different transfer functions and damping systems). Journal of Vibration and Acoustics, 125, pp 398405, 2003.

7. Piezoelectric ceramics data book for designers (Morgan Electroceramics, 1999). 